\title{
Menopause Impacts Human Brain Structure, Connectivity, Energy Metabolism, and Amyloid-beta Deposition.
}

Lisa Mosconi ( $\sim$ lim2035@med.cornell.edu )

Weill Cornell Medicine

Valentina Berti

University of Florence

Jonathan Dyke

Weill Cornell Medicine

Eva Schelbaum

Weill Cornell Medicine

Steven Jett

Weill Cornell Medicine

Lacey Laughlin

Weill Cornell Medicine

Grace Jang

Weill Cornell Medicine

Aneela Rahman

Weill Cornell Medicine

Hollie Hristov

Weill Cornell Medicine

Sikly Pahlajani

Weill Cornell Medicine

Randolph Andrews

ADM Diagnostics

Dawn Matthews

ADM Diagnostics

Orli Etingin

Weill Cornell Medicine

Christine Ganzer

Hunter College

Mony de Leon

Weill Cornell Medicine

Richard Isaacson 
Weill Cornell Medicine

\section{Roberta Brinton}

University of Arizona

\section{Research Article}

Keywords: menopause transition (MT), human brain, pre-menopause, peri-menopause, post-menopause

Posted Date: January 19th, 2021

DOl: https://doi.org/10.21203/rs.3.rs-146141/v1

License: (c) (1) This work is licensed under a Creative Commons Attribution 4.0 International License. Read Full License 


\section{Abstract}

All women undergo the menopause transition (MT) in their lifetime, yet little is known of how this process impacts the human brain. The MT occurs in stages characterized by unique endocrine properties that impact aging trajectories of multiple organ systems including brain. This multi-modality neuroimaging study indicates that MT stages (pre-menopause, peri-menopause, and post-menopause) are associated with substantial differences in brain structure, connectivity, and energy metabolism in regions subserving higher-order cognitive processes. Effects were specific to endocrine aging (menopause) rather than chronological aging, as determined by comparison to age-matched males. Brain biomarkers largely stabilized post-menopause, and gray matter volume (GMV) recovered in key brain regions sensitive to cognitive aging. Notably, GMV recovery and in vivo brain mitochondria ATP production correlated with preservation of cognitive performance post-menopause, suggesting adaptive compensatory processes. In parallel to the adaptive process, amyloid- $\beta$ deposition was more pronounced in peri-menopausal and post-menopausal women carrying apolipoprotein E-4 (APOE-4) genotype, the major genetic risk factor for late-onset Alzheimer's disease, relative to genotype-matched males. These data show that human menopause is a dynamic neurological transition that significantly impacts brain structure, connectivity, and metabolic profile, as well as cognitive integrity, during midlife endocrine aging of the female brain.

\section{Introduction}

The menopause transition (MT) is a midlife neuroendocrine aging process specific to females that culminates with reproductive senescence ${ }^{1}$. All women undergo menopause in their lifetime either through the natural endocrine aging process or through medical intervention. While menopause is a reproductive transition state, it is also a neurological transition ${ }^{1}$. As such, many of the symptoms of menopause are neurological in nature, such as hot flashes, disturbed sleep, mood changes, and forgetfulness ${ }^{2}$.

The MT is orchestrated by gonadal sex steroid hormones, which are known regulators of both reproduction and neural function ${ }^{1}$. During the MT, sex hormones, especially $17 \beta$-estradiol, substantially decline in body and brain ${ }^{3}$. Molecular, cellular and systems biology characterization of the MT in brain demonstrate a broad impact of estrogen declines on neural processes via genomic and non-genomic actions ${ }^{1}$, including changes in spinogenesis and synaptogenesis, neuronal number and morphology, glucose metabolism, and gene expression ${ }^{1,3}$. Additionally, estrogen depletion has been linked to amyloidbeta $(A \beta)$ plaque accumulation, a hallmark of Alzheimer's disease $(A D)$, in female animals ${ }^{4,5}$. However, little is known of how the MT impacts the human brain.

Brain imaging analyses conducted in postmenopausal women first reported declines in glucose metabolism and gray matter volume (GMV), which were modulated by menopause hormone treatment $(\mathrm{HT})$ (for review, see $\mathrm{e}^{6,7}$ ). These findings indicated that neuro-endocrine processes retain dynamic properties well into menopause. However, data in women undergoing the MT are scarce. We previously reported that, among women with genetic risk factors for $A D$ such as apolipoprotein epsilon- 4 genotype 
$(\mathrm{APOE}-4)^{8}$, the MT was associated with hypometabolism, lower GMV, and emerging A $\beta$ deposition ${ }^{9-11}$. It is unknown whether similar changes occur in women without genetic risks for AD. Analysis of brain structural, bioenergetic and cognitive integrity across MT is critical to understanding brain aging in women.

Herein, we conducted a multi-modality neuroimaging study of women at different MT stages (pre-, peri-, and post-menopause) to investigate MT's effects on brain's gray and white matter structure, connectivity, energy metabolism, and $A \beta$ deposition. Results indicate that the MT significantly impacts all these brain biomarkers in regions involved in higher-order cognitive functions. These effects were independent of age and HT use, and were specific to menopausal endocrine aging rather than chronological aging, as determined by comparison with age-matched males. Notably, cognition was preserved post-menopause, which correlated with GM volume recovery and brain adenosine triphosphate (ATP) production, highlighting potential compensatory mechanisms. Finally, $A \beta$ deposition was greater in post-menopausal and peri-menopausal women carrying APOE-4 genotype, indicating APOE-4 specific effects on AD risk with onset in the perimenopause.

\section{Results}

\section{Participants}

A total of 182 cognitively normal 40-65 year-old women were enrolled. Nine participants were excluded due to neuropathological conditions encountered in the MR images $(n=7$; e.g. neoplastic condition, aneurysm), or due to artifacts $(n=2)$. Three participants with incomplete clinical data and 9 with unclear menopause status were also excluded. Our final sample consisted of 161 women, including 30 premenopausal (PRE), 57 peri-menopausal (PERI), and 74 post-menopausal (POST) participants. There were no group differences in demographic or cognitive measures except for an age difference between PRE and POST groups (Supporting Information Table 1). Our procedures to address age effects are described in the Methods, and included comparing each MT group to an age-matched male group: 30 males agematched to PRE (MALE $\left.E_{P R E}\right), 50$ age-matched to PERI (MALE $E_{P E R I}$ ), and 45 age-matched to POST (MALE $\mathrm{POST}_{\text {; }}$ Supporting Information Table 2). $42 \%$ of participants were APOE-4 positive, with comparable distributions between groups (Supporting Information Tables 1 and 2).

\section{Biomarker differences between MT groups}

We examined MT-stage effects on a panel of biomarkers examining brain (a) structure: MRI-based gray and white matter volume (GMV, WMV) and fractional anisotropy (FA, an index of WM integrity and structural connectivity ${ }^{12}$ ); (b) energy metabolism: ${ }^{18}$ F-FDG PET glucose metabolism (CMRglc), ASL cerebral blood flow (CBF), and ${ }^{31}$ P-MRS ATP production; and (c) ${ }^{11} \mathrm{C}$-PiB-PET A $\beta$ deposition. Unless otherwise specified, all results are significant at $p<0.05$, cluster-level corrected for family-wise type error (FWE), adjusted for age, APOE-4 status, and modality-specific confounders. 


\section{Structural biomarkers}

GMV: MT stage differences were observed in inferior temporal gyrus, precuneus, and fusiform gyrus of the right hemisphere (Fig. 1a). On post-hoc analysis, the temporal cluster reflected lower GMV in the POST group compared to the PRE group, while the precuneus and fusiform clusters reflected lower GMV in the PERI group compared to the POST group (Fig. 1b and Supporting Information Table 3).

WMV: Significant MT stage differences were observed in anterior and posterior corona radiata (Fig. 1c). On post-hoc analysis, these clusters reflected lower WMV in the POST group vs. PRE and PERI groups (Fig. 1d and Supporting Information Table 4).

FA: The only region that survived FWE correction was a cluster of lower FA in the right external capsule of POST compared to PERI (Fig. 1e, f, g, and Supporting Information Table 5).

\section{Metabolic biomarkers}

CMRglc: Significant MT stage differences were observed in supramarginal gyrus, middle and inferior temporal gyri (Fig. 2a). On post-hoc analysis, all clusters reflected lower CMRglc in POST vs. PRE and PERI groups (Fig. 2b and Supporting Information Table 6). The PERI group also showed lower CMRglc than PRE in right middle temporal gyrus (Fig. 2b and Supporting Information Table 6).

CBF: Significant MT stage differences were observed in supramarginal gyrus, middle and superior temporal gyrus, superior and inferior frontal gyrus (Fig. 2c). On post-hoc analysis, these clusters reflected higher CBF in POST vs. PERI (Fig. 2d and Supporting Information Table 7). Figure 2e displays the regional overlap of CMRglc and CBF effects.

ATP production: We examined regional ATP to phosphocreatine ( $\mathrm{PCr}$ ) ratios in parieto-temporal regions showing MT-stage effects on CMRglc and CBF (Table 1). Multivariate general linear models (mGLM) revealed significant ATP/PCr group differences (Wilk's Lambda $p=0.030$ ), which on post-hoc examination, were driven by POST exhibiting higher ATP/PCr in temporal regions ( $p$ 's $<0.047$ ) and borderline higher ATP/PCr in parietal regions ( $p$ 's $\leq 0.12$ ) compared to PRE. The PERI group showed intermediate ATP/PCr levels. Results were unchanged including APOE status as a covariate (Table 1). 
Table 1

${ }^{31} \mathrm{P}-\mathrm{MRS}$ ATP/PCr measures by menopausal stage group

\begin{tabular}{|c|c|c|c|}
\hline & $\begin{array}{l}\text { Pre-menopausal } \\
\text { group }\end{array}$ & $\begin{array}{l}\text { Peri-menopausal } \\
\text { group }\end{array}$ & $\begin{array}{l}\text { Post-menopausal } \\
\text { group }\end{array}$ \\
\hline $\begin{array}{l}\text { Superior temporal } \\
\text { gyrus }\end{array}$ & $1.11(0.05)$ & $1.18(0.03)$ & $1.26(0.03)^{\star}$ \\
\hline $\begin{array}{l}\text { Adjusted by } A P O E \\
\text { status }\end{array}$ & $1.14(0.04)$ & $1.18(0.03)$ & $1.24(0.02)^{\star}$ \\
\hline Middle temporal gyrus & $1.09(0.04)$ & $1.14(0.02)$ & $1.20(0.02)^{\star}$ \\
\hline $\begin{array}{l}\text { Adjusted by APOE } \\
\text { status }\end{array}$ & $1.09(0.03)$ & $1.13(0.02)$ & $1.19(0.02)^{\star}$ \\
\hline Inferior temporal gyrus & $1.07(0.04)$ & $1.11(0.02)$ & $1.13(0.02)^{\star}$ \\
\hline $\begin{array}{l}\text { Adjusted by } A P O E \\
\text { status }\end{array}$ & $1.07(0.03)$ & $1.10(0.02)$ & $1.14(0.02)^{\star}$ \\
\hline Inferior parietal lobule & $0.77(0.06)$ & $0.76(0.05)$ & $0.83(0.04)$ \\
\hline $\begin{array}{l}\text { Adjusted by APOE } \\
\text { status }\end{array}$ & $0.78(0.04)$ & $0.75(0.03)$ & $0.83(0.03)^{*}$ \\
\hline
\end{tabular}

\section{Amyloid- $\beta$ load}

There were no significant differences in PiB uptake between MT groups (Table 2). However, adding APOE4 status (positive vs. negative) as a covariate enhanced group differences, which reached significance for POST vs. PRE (mean difference, $27 \%, p=0.005)$, and for PERI vs. PRE groups $(18 \%, p=0.048)$. 
Table 2

PiB-PET amyloid- $\beta$ load by menopausal stage group

\begin{tabular}{|c|c|c|c|}
\hline & $\begin{array}{l}\text { Pre-menopausal } \\
\text { group }\end{array}$ & $\begin{array}{l}\text { Peri-menopausal } \\
\text { group }\end{array}$ & $\begin{array}{l}\text { Post-menopausal } \\
\text { group }\end{array}$ \\
\hline AD-mask SUVR & $1.03(0.12)$ & $1.20(0.06)$ & $1.27(0.07)$ \\
\hline $\begin{array}{l}\text { adjusted by } A P O E \\
\text { status }\end{array}$ & $1.00(0.09)$ & $1.21(0.06)$ & $1.29(0.06)^{*}$ \\
\hline \multicolumn{4}{|c|}{ AD-mask SUVR by APOE-4 status } \\
\hline APOE-4- & $0.94(0.12)$ & $1.12(0.07)$ & $1.32(0.07)^{\star}$ \\
\hline APOE-4+ & $1.07(0.12)$ & $1.39(0.11)^{\star}$ & $1.25(0.08)^{\star}$ \\
\hline PiB scans, $\%$ positive & 5 & 16 & $24^{\star}$ \\
\hline \multicolumn{4}{|c|}{ PiB scans, \% positive by APOE- 4 status } \\
\hline APOE-4- & 0 & 10 & 22 \\
\hline APOE-4+ & 9 & $31^{*}$ & $26^{*}$ \\
\hline \multicolumn{4}{|c|}{$\begin{array}{l}\text { Values are age-adjusted means (SE), unless otherwise specified. *Different from pre-menopausal } \\
\text { group, } p<0.05 \text {. }\end{array}$} \\
\hline \multicolumn{4}{|c|}{$\begin{array}{l}\text { Abbreviations: APOE-4-, APOE-4 non-carriers; APOE-4+, APOE-4 carriers; SUVR, standardized uptake } \\
\text { value ratio to cerebellar gray matter PiB uptake. }\end{array}$} \\
\hline
\end{tabular}

Twenty (18\%) female participants undergoing PiB-PET imaging were brain amyloid positive. The frequency of PiB-positive scans was borderline higher in POST vs. PRE (24\% vs. 5\%; Pearson Chi Square $p=0.056)$, and intermediate in PERI (16\%, n.s.; Table 2). Among APOE-4 carriers, the frequency of PiBpositive scans was higher in POST (26\%) and PERI (31\%) vs. PRE ( $9 \%$; Pearson Chi Square p $=0.049$ ). Among non-carriers, the frequency of PiB-positive scans was highest in POST (22\%), intermediate in PERI (10\%), and lowest in PRE (0\%), which did not reach significance.

\section{Comparisons to age-matched males}

Unless otherwise specified, all results are significant at $p<0.05$, cluster-level FWE corrected, adjusted for modality-specific confounders.

\section{Structural biomarkers}

GMV: Results are presented in Fig. 3a and Supporting Information Table 8. Analyses confirmed presence of lower GMV in inferior temporal gyrus of the POST group vs. MALE $E_{P O S T}$, and of lower GMV in precuneus and fusiform gyrus of the PERI group vs. MALE $E_{P E R I}$. In the entire search volume, both POST and PERI exhibited widespread areas of lower GMV compared to males, involving inferior, middle and medial 
temporal (MTL) regions, fusiform gyrus, superior, middle, and orbital frontal gyrus, anterior cingulate (ACC), insula, and putamen. Additionally, the PERI group exhibited lower GMV in precuneus vs. MALE $E_{\text {PERI }}$. The PRE group showed less extended areas of lower GMV compared to vs. MALE $E_{P R E}$ involving MTL, frontal gyrus, putamen, and temporal cortex.

WMV: Results are presented in Fig. 3b and Supporting Information Table 9. Analyses confirmed presence of lower WMV in corona radiata of the POST group vs. MALE $E_{\text {POST, }}$ and revealed additional areas of lower WMV in several tracts (Fig. 3b). The PERI group exhibited a similar pattern of lower WMV compared to vs. $M_{A L E} E_{P E R}$, and the PRE group showed lower WMV in internal capsule vs. MALE ${ }_{P R E}$.

FA: Results are presented in Fig. 3c and Supporting Information Table 10. The POST group exhibited no regions of lower FA vs. MALE $E_{P O S T}$, and showed instead higher FA in corona radiata and fornix. The PERI group exhibited higher FA than MALE $E_{P E R I}$ in corona radiata, fornix, external capsule and uncinate fasciculus; and lower FA in superior longitudinal fasciculus (SFL) and posterior thalamic radiation. The PRE group also showed higher FA in corona radiata, and lower FA in SLF, compared to vs. MALE $E_{P R E}$.

\section{Metabolic biomarkers}

CMRglc: Results are presented in Fig. 3d and Supporting Information Table 11. Analyses confirmed presence of lower CMRglc in temporo-parietal areas of the POST group vs. MALE $E_{\mathrm{POST}}$, and revealed additional areas of hypometabolism in superior and middle frontal cortex. The PERI group showed lower CMRglc in frontal cortex and insula vs. MALE $E_{P E R l}$ and the PRE group showed lower CMRglc in left supramarginal gyrus vs. MALE $E_{\mathrm{PRE}}$. All results were significant at $p<0.05$, FWE-corrected.

CBF: Results are presented in Fig. 3e and Supporting Information Table 12. Analyses confirmed presence of elevated CBF in frontal regions of the POST group, and revealed areas of lower CBF in ACC, MTL, and basal ganglia vs. MALE $E_{P O S T}$. The PERI and PRE groups also had areas of higher and lower CBF compared to males. Both groups exhibited lower CBF than males in ACC, MTL, superior temporal gyrus, and basal ganglia, and higher $\mathrm{CBF}$ in parietal lobe. All results were significant at $\mathrm{p}<0.05$, FWE-corrected.

ATP production: We examined ATP/PCr using two separate mGLMs: model 1 included regions with higher

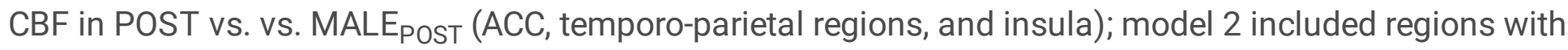

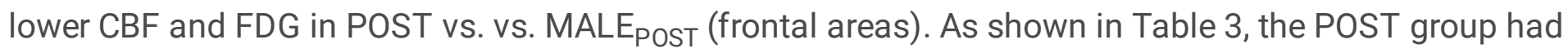
higher ATP/PCr than MALE POST $_{\text {in }}$ model 1 (Wilk's Lambda $p=0.003$, APOE-adjusted $p=0.002$ ) and borderline higher ATP/PCr in model 2 (Wilk's Lambda $p=0.062$, APOE-adjusted $p=0.082$ ). The PERI

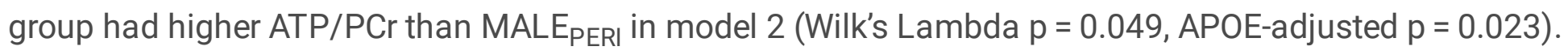
There were no differences between PRE and vs. MALE $E_{P R E}$. 
Table 3

MRS ATP/PCr measures by age-matched group comparisons

\begin{tabular}{|c|c|c|c|c|c|c|}
\hline & $\begin{array}{l}\text { Pre- } \\
\text { menopausal } \\
\text { group }\end{array}$ & $\begin{array}{l}\text { Age- } \\
\text { matched } \\
\text { males }\end{array}$ & $\begin{array}{l}\text { Peri- } \\
\text { menopausal } \\
\text { group }\end{array}$ & $\begin{array}{l}\text { Age- } \\
\text { matched } \\
\text { males }\end{array}$ & $\begin{array}{l}\text { Post- } \\
\text { menopausal } \\
\text { group }\end{array}$ & $\begin{array}{l}\text { Age- } \\
\text { matched } \\
\text { males }\end{array}$ \\
\hline \multicolumn{7}{|l|}{$\underset{1}{\text { Model }}$} \\
\hline ACC & $1.06(0.07)$ & $1.11(0.07)$ & $1.10(0.04)$ & $1.12(0.05)$ & $1.13(0.04)$ & $1.12(0.05)$ \\
\hline Insula & $1.21(0.06)$ & $1.29(0.07)$ & $1.23(0.04)$ & $1.24(0.04)$ & $1.26(0.03)^{\star}$ & $1.20(0.05)$ \\
\hline IPL & $0.78(0.07)$ & $0.85(0.08)$ & $0.76(0.04)$ & $0.84(0.04)$ & $0.86(0.03)$ & $0.83(0.04)$ \\
\hline MiTG & $1.10(0.04)$ & $1.17(0.05)$ & $1.15(0.02)$ & $1.12(0.3)$ & $1.19(0.02)^{\star}$ & $1.10(0.03)$ \\
\hline MTL & $1.16(0.05)$ & $1.24(0.05)$ & $1.22(0.03)$ & $1.20(0.04)$ & $1.21(0.03)$ & $1.19(0.04)$ \\
\hline \multicolumn{7}{|l|}{$\begin{array}{l}\text { Model } \\
2\end{array}$} \\
\hline MFG & $1.05(0.06)$ & $0.99(0.06)$ & $1.04(0.03)$ & $0.98(0.04)$ & $1.07(0.03)^{\star}$ & $0.95(0.04)$ \\
\hline SFG & $0.95(0.05)$ & $0.86(0.06)$ & $0.94(0.03)^{\star}$ & $0.84(0.03)$ & $0.96(0.03)^{\star}$ & $0.82(0.04)$ \\
\hline \multicolumn{7}{|c|}{ Values are means (SE). *Different from age-matched males on univariate post-hoc analysis, $p<0.05$. } \\
\hline $\begin{array}{l}\text { Abbrev } \\
\text { MiTG, } 1\end{array}$ & $\begin{array}{l}\text { Ions: ACC, ant } \\
\text { Idle temporal }\end{array}$ & $\begin{array}{l}\text { or cingulate } \\
\text { us; MTL, m }\end{array}$ & $\begin{array}{l}\text { rtex; IPL, infer } \\
\text { al temporal lo }\end{array}$ & $\begin{array}{l}\text { parietal lob } \\
\text { SFG, superi }\end{array}$ & $\begin{array}{l}\text { MFG, medial } \\
\text { frontal gyrus. }\end{array}$ & tal gyrus; \\
\hline
\end{tabular}

\section{Amyloid- $\beta$ load}

POST and PERI groups exhibited higher PiB uptake in AD-mask than the corresponding male groups (p's $\leq 0.001$; Table 4), while no differences were found between PRE and MALE $E_{P R E}$. These effects remained unchanged after including APOE status as a covariate, which nonetheless enhanced differences between PERI and MALE $E_{P E R I}$ APOE-4 carriers $(p=0.020)$. 
Table 4

PiB-PET amyloid- $\beta$ load by age-matched group comparisons

\begin{tabular}{|c|c|c|c|c|c|c|}
\hline & $\begin{array}{l}\text { Pre- } \\
\text { menopausal } \\
\text { group }\end{array}$ & $\begin{array}{l}\text { Age- } \\
\text { matched } \\
\text { males }\end{array}$ & $\begin{array}{l}\text { Peri- } \\
\text { menopausal } \\
\text { group }\end{array}$ & $\begin{array}{l}\text { Age- } \\
\text { matched } \\
\text { males }\end{array}$ & $\begin{array}{l}\text { Post- } \\
\text { menopausal } \\
\text { group }\end{array}$ & $\begin{array}{l}\text { Age- } \\
\text { matched } \\
\text { males }\end{array}$ \\
\hline $\begin{array}{l}\text { AD-mask } \\
\text { SUVR }\end{array}$ & $1.01(0.05)$ & $0.92(0.05)$ & $1.20(0.05)^{\star}$ & $0.92(0.06)$ & $1.29(0.05)^{\star}$ & $0.93(0.06)$ \\
\hline $\begin{array}{l}\text { adjusted by } \\
\text { APOE-4 } \\
\text { status }\end{array}$ & $1.01(0.05)$ & $0.92(0.05)$ & $1.20(0.05)^{*}$ & $0.91(0.06)$ & $1.29(0.05) *$ & $0.93(0.06)$ \\
\hline \multicolumn{7}{|c|}{ AD-mask SUVR by APOE-4 status } \\
\hline APOE-4- & $0.94(0.07)$ & $0.86(0.07)$ & $1.12(0.06)$ & $0.88(0.08)$ & $1.32(0.07) *$ & $0.93(0.09)$ \\
\hline APOE-4+ & $1.07(0.07)$ & $0.96(0.07)$ & $1.39(0.09)^{\star}$ & $0.96(0.08)$ & $1.26(0.07)^{\star}$ & $0.95(0.10)$ \\
\hline $\begin{array}{l}\text { PiB scans, \% } \\
\text { positive }\end{array}$ & 5 & 0 & $16^{*}$ & 0 & $24^{\star}$ & 3 \\
\hline \multicolumn{7}{|c|}{ PiB scans, $\%$ positive by APOE- 4 status } \\
\hline APOE-4- & 0 & 0 & 10 & 0 & 22 & 6 \\
\hline APOE-4+ & 9 & 0 & $31^{*}$ & 0 & $26^{*}$ & 0 \\
\hline \multicolumn{7}{|c|}{ Values are age-adjusted means (SE). *Different from age-matched males, $p<0.05$. } \\
\hline \multicolumn{7}{|c|}{ Abbreviations: see legend to Table 2 . } \\
\hline
\end{tabular}

The frequency of PiB-positive scans was higher in POST vs. MALE $0.015)$, and in PERI vs. MALE $E_{\text {PERI }}(16 \%$ vs. $0 \%$, respectively, $p=0.012)$. An effect of APOE-4 status was noted, with POST and PERI carriers exhibiting greater frequency of PiB-positive scans than male carriers ( $p$ 's $<0.050$ ), whereas no differences were found among non-carriers. There were no differences in PiBpositivity for PRE vs. MALE $E_{P R E}$, independent of APOE status.

\section{Biomarker changes post-menopause}

To examine whether biomarker effects were persistent post-menopause, and to test their specificity for menopause, we performed 2-year follow-up volumetric MRI and FDG-PET scans in a subset of 17 POST women and 12 MALE $_{\text {POST }}$ (Supporting Information Table 13). All results are significant at $p<0.05$, clusterlevel FWE corrected, adjusted for time to follow-up, APOE-4 status, and modality-specific confounders.

GMV: Results are presented in Fig. 4a and Supporting Information Table 14. There were no GMV changes in inferior temporal and fusiform gyri of the POST group, whereas GMV increases were observed in the precuneus. These changes were significant compared to MALE $E_{\text {POST }}$. 


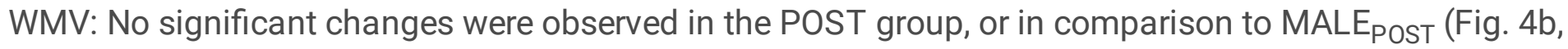
d).

CMRglc: Results are presented in Fig. 4c, d and Supporting Information Table 15. Within temporo-parietal regions that were hypometabolic in the POST group, CMRglc declines were observed only in left inferior parietal lobule, which were significant compared to MALE $E_{\mathrm{POST}}$. There were no further longitudinal differences between groups.

\section{Associations between biomarkers and cognition}

As shown in Supporting Information Table 16 and Supporting Information Fig. 1, GMV in precuneus was positively associated with memory scores cross-sectionally (Pearson's $r=0.311, p=0.007$ ) and longitudinally (Pearson's $r=0.586, p=0.013$ ). The rate of GMV increase in precuneus was also positively associated with memory performance (Pearson's $r=0.521, p=0.032$ ). ATP/PCr measures in temporal regions were positively associated with global cognition (Spearman's Rho $=0.329, p=0.012$ ).

\section{Menopausal hormone therapy (HT) and hysterectomy status}

We performed a sensitivity analysis to examine the effects of HT and hysterectomy status on brain biomarkers, included in Supporting Information Appendix. Briefly, including these variables as covariates left MT effects substantially unchanged. Restricting analysis to HT non-users confirmed results from the entire cohort, with two exceptions: CBF differences between groups were more pronounced among nonusers, and FA differences between PERI and POST did not reach significance. As compared to HT users, non-users exhibited lower GMV in inferior temporal gyrus, and lower CMRglc in parietal regions, independent of hysterectomy status.

\section{Discussion}

This multi-modality neuroimaging study demonstrates that MT stage has pronounced effects on human brain's structure, connectivity, and energy metabolism, which provide a neurological framework for both vulnerability and resilience. Effects were most pronounced in estrogen-regulated networks subserving cognitive processes, and were independent of age, APOE-4 status, HT usage, and hysterectomy status. Comparisons to age-matched males provided further evidence that biomarker effects were specific to endocrine menopausal aging rather than chronological aging. In most brain regions and across modalities, brain biomarkers stabilized or recovered post-menopause. Cognitive preservation postmenopause correlated with GMV recovery and brain ATP production, highlighting cognitive recovery mechanisms. In parallel to the adaptive process, A $\beta$ deposition was more pronounced in PERI and POST women positive for APOE-4 genotype, indicating specificity for AD with onset in the perimenopause. 
The MT is a normal physiological event. However, while the majority of women undergo menopause without long-term adverse effects ${ }^{1}$, many are vulnerable to the neurological shifts that can occur during this transition and experience bothersome symptoms ${ }^{2}$, as well as a higher risk of depression, anxiety, and $A D^{13}$. Preclinical work indicates that the brain has the ability to compensate for changes in estrogen levels and receptor activity during $\mathrm{MT}^{1}$. In some instances, however, compensatory responses are diminished, lacking, or limited to some estrogen-regulated networks ${ }^{1}$, which may account for the complex MT phenotype observed in women ${ }^{2}$. While investigation of these compensatory responses is still in the early stages ${ }^{1}$, it's been hypothesized that gradual hormonal changes during spontaneous MT may allow "brain resetting", e.g. neuronal adaptations to the hypo-estrogenic post-menopausal state ${ }^{1,14,15}$. Brain adaptation may account for the easing of menopausal symptoms like hot flashes, which tend to resolve 2-7 years into menopause ${ }^{2}$.

Present neuroimaging results provide novel neurophysiological evidence for post-menopausal brain adaptation in humans, encompassing brain structure, connectivity and bioenergetics, and preservation of cognitive function.

Compared to age-matched males, POST and PERI groups exhibited lower GMV in widespread cortical areas including temporo-parietal, frontal, cingulate, and insular cortex, as well as subcortical structures such as hippocampus, amygdala, and thalamus. However, GMV largely stabilized post-menopause and recovered in precuneus, an associative cortical area involved in social processes, episodic memory, and information integration ${ }^{16}$. On average, in the POST group, GMV in precuneus (a) was higher than the PERI group, (b) was comparable to age-matched males, and (c) showed increases at the 2-year follow-up. Further, GMV in precuneus correlated with memory scores at cross-section and longitudinally, indicating that this area undergoes structural changes that are influenced by MT and have cognitive implications. Brain imaging studies of pregnancy (also a female-specific neuro-endocrine transition) reported dynamic GMV changes in the precuneus, which are thought to reflect reduced neurogenesis in late pregnancy, followed by restoration by the time of weaning ${ }^{17}$. Although neurogenesis decreases with aging ${ }^{18}$, similar mechanisms may play a role in the brain's adaptation to the post-menopausal stage. In fact, the observed MT-related anatomical pattern maps onto estrogen-regulated systems ${ }^{1}$ and displays notable similarities to the neural networks impacted by pregnancy ${ }^{19}$.

MT stage also influenced WMV within major tracts connecting extensive parts of the cerebral cortex and subcortical regions. Both POST and PERI groups exhibited widespread WMV loss compared to agematched males, with the POST group also exhibiting lower WMV in corona radiata compared to PRE and PERI groups. In spite of the WMV loss, however, all MT groups exhibited higher FA than males in corona radiata, as well as in fornix of the POST group, and in fornix, uncinate fasciculus and external capsule of the PERI group. PERI and PRE groups also exhibited some clusters with lower FA than males, mostly involving SLF, whereas the POST group showed no deficits. While there are no previous DTI studies of MT, there is evidence for sex differences in WM microstructure that vary with age and neuro-endocrine status. Most DTI studies on the entire age range found that males have generally higher FA than females in WM 
tracts such as SLF ${ }^{20}$ - as in our comparisons with PERI and PRE groups. In contrast, during puberty (the first neuro-endocrine transition stage for both genders), females display higher FA than males in several WM tracts including corona radiata and fornix ${ }^{21}$. Animal studies report that pregnancy is also accompanied by increased regional water diffusivity ${ }^{22}$. While FA has a number of determinants, the principle factors are myelination and tissue architecture, with the inference that higher FA represents more "efficient" WM organization ${ }^{12}$. As such, our findings suggest greater efficiency of a smaller corona radiata and fornix post-menopause, raising the possibility that MT is accompanied by further refinement of these regions' connectivity.

The MT also impacted brain energetics on multiple levels. The POST group, and to a lesser extent the PERI group, exhibited hypometabolism in parieto-temporal cortices, consistent with previous reports in women at risk for $A D^{9-11}$. However, in the present study, regional CMRglc largely plateaued postmenopause, suggesting adaptation to a new metabolic baseline after prolonged estrogen deficiency. Additionally, CBF and ATP production in temporo-parietal regions were higher post-menopause, and ATP levels positively correlated with global cognition. Findings of higher CBF in women are consistent with the literature ${ }^{23}$, and indicate that MT modulates CBF sex differences in midlife. To our knowledge, there are no previous studies of MT on brain ATP production in humans. Altogether, present results provide novel evidence for in vivo neurovascular-neurometabolic dissociations during MT. Typically, regional brain activity, $\mathrm{CMRglc}$, and $\mathrm{CBF}$ are coupled ${ }^{24}$. Dissociation can occur with aging, pathology, and inflammation ${ }^{24}$ - or as a compensatory response ${ }^{25}$. While data in humans are scarce, preclinical evidence indicates that estrogen loss during MT triggers CMRglc declines ${ }^{26}$, prompting an adaptive reaction to increase ketone bodies utilization as an alternative fuel for ATP ${ }^{27}$. Continued reliance on ketones leads to compromised mitochondrial function, WM catabolism, and cellular apoptosis in animals $^{26,27}$. Our neuroimaging findings confirm presence of hypometabolism during MT, and suggest that the observed higher CBF and ATP levels in POST women reflect a compensatory reaction to glucose deprivation, as well as a means to increase ketone metabolism.

We previously reported reduced peripheral mitochondrial cytochrome oxidase activity (COX) in POST women at risk for $A D^{28}$. Herein, brain ATP production was higher in POST women independent of APOE-4 status, suggesting different mitochondrial activity profiles in brain and periphery. Animal models of menopause also show dynamic relationships between central and peripheral metabolic systems, which tend to shift from uncoupled to coupled under metabolic stress ${ }^{29}$. Although brain mitochondria might eventually falter in older POST women, the positive associations between ATP production and cognition in midlife suggest a recovery mechanism instead. As aging and menopause differ to some extent between humans and animals, the neuro-energetics of menopause are also likely to differ- in this case, by supporting women's ability to transition into late life with preserved cognition.

In our study, POST women did not exhibit reduced cognitive performance as compared to the other groups. While self-reports of poor memory and concentration are common in women undergoing $\mathrm{MT}^{2}$, menopause itself hasn't been associated with deficits on cognitive testing ${ }^{30}$. Further, it is well- 
documented that women perform better than men on several cognitive domains across the adult lifespan- an advantage that seems to persist even after a dementia diagnosis ${ }^{31}$. Nonetheless, our sample was highly educated and results may not apply to women of diverse background. More ecologically valid tests might also reveal subtle or transitory changes associated with MT.

Consistent with previous studies of MT women at risk for $A D^{9-11}$, our POST and PERI APOE-4 carriers exhibited higher $A \beta$ deposition compared to age-matched males. While $A \beta$ deposition was mild, these data support evidence that interactions between age, female gender and APOE-4 increase AD vulnerability during perimenopause ${ }^{32}$. Chronologically, MT maps onto the preclinical phase of $A D$, which begins decades prior to symptom onset ${ }^{33}$. The earlier onset of, and longer exposure to $A \beta$ pathology may account for the higher prevalence of $A D$ in females, with POST women comprising almost two thirds of all cases ${ }^{34}$. It warrants emphasis that reproductive aging is not uniformly associated with AD risk. In our cohort, $18 \%$ of women undergoing PiB-PET were brain A $\beta$-positive. While some of these women might develop $A D$, for others, $A \beta$ deposition could reflect accelerated biological aging due to hormonal declines instead $^{35}$. In fact, over $20 \%$ of healthy elderly display moderate cerebral $A \beta$ burden and no dementia ${ }^{33}$.

The proportion of APOE-4 carriers in our study was $42 \%$, which is higher than the $15-30 \%$ observed in the general population ${ }^{36}$. Many of our participants volunteer because of concerns about cognitive functioning, which tend to be more frequent among APOE-4 carriers ${ }^{37}$. As such, our cohort may be selfenriched with individuals at higher a priori risk of AD. Although our results were independent of APOE-4 status, more studies are needed to replicate these findings in community-dwelling individuals randomly recruited from the population.

The observed MT effects were independent of HT and hysterectomy status, though HT use was associated with mild beneficial effects on GMV and CMRglc, consistent with some clinical trials ${ }^{6}$. Whether $\mathrm{HT}$ provides protection against cognitive aging and $\mathrm{AD}$ remains however unclear. Observational studies generally found positive effects on cognition across multiple HT regimens ${ }^{38}$, whereas clinical trials reported an increased dementia risk with estrogen-plus-progestin $\mathrm{HT}$, and no effects with estrogenalone, in late POST women 65 or older $\mathrm{HT}^{39}$. No adverse or beneficial effects on cognition were observed in early POST women ${ }^{40,41}$. Overall, HT's efficacy is thought to depend on timing of treatment initiation with respect to age at menopause, with benefits pertaining to early initiation, especially after induced menopause ${ }^{7,39}$. Our neuroimaging results point to the MT as a dynamic neurological process and, therefore, a window of vulnerabilities and opportunities when the human brain is influenced by biochemical adjustments, but is also receptive to interventions. More studies are needed to test the efficacy of HT instituted prior to menopause.

From a methodological perspective, we examined statistically powered groups of women at different MT stages, paired with age correction procedures including comparisons to age-matched males and longitudinal evaluations in a subset of participants. Nonetheless, a causal link between MT and brain biomarkers cannot be unequivocally established. We chose this study design because the timing of 
menopause is highly variable, with a median age at menopause of 51 years, and a distribution of $40-58$ years $^{2}$. Longitudinal studies may require 10-15 years of follow-ups to capture the effects of the MT on brain biomarkers. While studies of induced (surgical) menopause ideally reduce follow-up times, the procedure may be associated with different, possibly more severe outcomes ${ }^{42,43}$. Future longitudinal studies are warranted to replicate our findings and test for differential effects of induced and spontaneous menopause.

Determination of MT status was based on established diagnostic criteria in accord with clinical and laboratory findings ${ }^{44}$. Nonetheless, some PERI women may have been early POST, while some early POST women could have been still late PERI. This would, however, conservatively reduce power in detecting MT stage differences. Considering the hormonal changes women experience during MT, and the extent of the observed biomarker effects, we attribute our results to the endocrine climate of menopause. Additionally, males of similar demographic characteristics were examined to account for other clinical and lifestyle variables associated with brain aging ${ }^{13}$. Future studies tracking changes in hormones, medical status, environment and lifestyle are needed to clarify the complex relationships between MT and brain aging, and identify which factors impact successful vs. unsuccessful recovery.

In evolutionary biology, there is active debate over whether human menopause is an adaptive process. In most animals species, females have the capacity to live beyond reproduction but rarely do. Many hypotheses exist about why human menopause evolved, some of which consider it a result of chance or of humans' longer lifespan, while others hypothesize an adaptive mechanism (for review, see ${ }^{45}$ ). According to the leading adaptive theory, the "grandmother hypothesis", programmed ovarian failure favors women who become infertile years before death and help to care for existing children and grandchildren instead of producing more ${ }^{46}$. These women may have been granted indirect reproductive fitness by manifesting a reproduction senescence phenotype encoded in late-onset fertility-diminishing mutation genotypes still present today ${ }^{47}$. From a neurological perspective, humans have evolved under different evolutionary pressures than other animal species, which put development of higher cognitive skills in a critical role. Our neuroimaging findings provide neurophysiological support to evolutionary conceptualizations of MT being an adaptive process in humans.

Overall, present findings show that human menopause is a dynamic neurological transition that reshapes the neural landscape of the female brain during midlife endocrine aging, and provide preliminary evidence for an adaptive process serving the transition into late life.

\section{Methods}

\section{Participants}

This is a non-randomized natural history non-treatment study of healthy, cognitively normal female participants ages 40-65 years at different menopausal stages, as well as male participants in the same age range, recruited at Weill Cornell Medicine and NYU School of Medicine between 2015-2020. 
Participants were recruited by self-referral, flyers, and word of mouth, as described ${ }^{9-11}$. Pre-established exclusion criteria included medical conditions that may affect brain structure or function (e.g. stroke, any neurodegenerative diseases, major psychiatric disorders, hydrocephalus, intracranial mass and infarcts on MRI), use of psychoactive medications, and contraindications to MRI or PET. Participants had Mini Mental Status Exam $\geq 27$ and normal cognitive performance for age and education ${ }^{9-11}$.

The patients' sex was determined by self-report. APOE genotype was assessed using standard qPCR procedures $^{9-11}$. Participants carrying one or two copies of APOE-4 allele were grouped as carriers, and compared to non-carriers.

\section{Standard protocol approvals, registrations, and patient consents}

All methods were carried out in accordance with relevant guidelines and regulations. All experimental protocols were approved by the Weill Cornell Medicine and NYU School of Medicine Institutional Review Boards. Written informed consent was obtained from all participants.

\section{Cognitive testing}

Our neuropsychological testing battery included tests measuring three cognitive domains: memory (immediate and delayed recall of a paragraph and paired associates), higher-order processing (block design tests), and language (object naming) ${ }^{9-11}$. We computed (i) a global cognitive score by creating Zscores within each domain and averaging across the domains; and (ii) a memory score by creating Zscores for each memory test and averaging across tests. The Kruskal-Wallis non-parametric test was used to examine global cognition between groups as this measure did not follow a normal distribution. GLMs with post-hoc Sidak tests were used to examine memory scores.

\section{Menopause assessments}

Determination of menopausal status was based on the Stages of Reproductive Aging Workshop (STRAW) criteria ${ }^{44}$ and corroborated by means of hormone assessments. Female participants were classified as regular cyclers (PRE), irregular cyclers (PERI), and no cycle for 12 or more months (POST).

\section{Brain Imaging Acquisition and Analysis}

All participants underwent structural MRI, and 160 participants received ${ }^{18} \mathrm{~F}$-Fluorodeoxyglucose (FDG) and ${ }^{11} \mathrm{C}$-Pittsburgh Compound B (PiB) PET at Weill Cornell Medicine. In 2017, we started acquiring DTI, 
ASL, and MRS scans, which have been done on 100 participants (Supporting Information Tables 18 and 19).

Our protocol included three MRI sequences on a 3.0 T G.E. Discovery MR750 scanner: (a) a sagittal T1

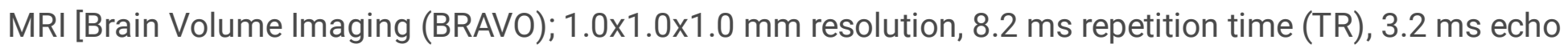
time (TE), $25.6 \mathrm{~cm}$ field of view (FOV), 256x256 matrix] used to estimate GMV and WMV; (b) a Diffusion Tensor Imaging (DTI) scan [55 directions, b-value = 1000s $/ \mathrm{mm}^{2}, 8000 \mathrm{~ms}$ TR, $65 \mathrm{~ms}$ TE, 256x256 matrix, $0.9 \times 0.9 \times 1.8 \mathrm{~mm}$ resolution] used to estimate $\mathrm{FA}^{12}$; (c) an Arterial Spin Labeling (ASL) scan [pseudocontinuous technique with $4851 \mathrm{~ms} \mathrm{TR}, 10.6 \mathrm{~ms} \mathrm{TE}, 4$ averages, $24 \mathrm{~cm}$ FOV, 2.0x2.0x3.8 mm resolution] used to estimate CBF (mL/100 $\mathrm{g} / \mathrm{min})$ using arterial blood water ${ }^{48}$.

${ }^{31}$ Phosphorus Magnetic Resonance Spectroscopy ( $\left.{ }^{31} \mathrm{P}-\mathrm{MRS}\right)$ was used to assess mitochondrial function in brain through the mapping of intracellular ATP and $\mathrm{PCr}$ levels ${ }^{49,50}$. A higher ATP/PCr ratio reflects greater ATP production relative to utilization ${ }^{51}$. MRS data was acquired on the GE scanner using a dual tuned 32-channel ${ }^{31} \mathrm{P} /{ }^{1} \mathrm{H}$ quadrature head coil [2048 points, $5000 \mathrm{~Hz}$ sweep width, $2000 \mathrm{~ms}$ TR, 2 averages, $55^{\circ}$ flip angle at $51.3 \mathrm{MHz}, 24 \mathrm{~cm}$ FOV]. Prior to ${ }^{31} \mathrm{P}-\mathrm{MRS}$, shimming was performed using a ${ }^{1} \mathrm{H}$ single voxel technique placed over the entire brain. MRS data was processed using XSOS written in IDL. Raw data was processed using Hamming and Fermi k-space filters, $20 \mathrm{~Hz}$ exponential filtering and zerofilling in time, $x$ and $y$-domains prior to 3D Fast Fourier Transformation. The PCr peak is set at $0.0 \mathrm{ppm}$ and susceptibility corrections performed. Baseline correction was applied by an experienced analyst (JPD). This resulted in a $16 \times 16$ image of $1.5 \times 1.5 \times 3.0 \mathrm{~cm}$ voxels with the signal intensity in each voxel corresponding to the peak area of the ${ }^{31} \mathrm{P}$ metabolite. The central $42 \mathrm{D}$ slices of CSI data were then registered to the BRAVO sequence.

FDG and PIB-PET scans were acquired on a Siemens BioGraph mCT 64-slice PET/CT operating in 3D mode [70 cm transverse FOV, $16.2 \mathrm{~cm}$ axial FOV], following standardized procedures ${ }^{9-11}$. Summed FDG and $\mathrm{PiB}$ images were obtained $40-60$ min post-injection of $5 \mathrm{mCi}$ of ${ }^{18} \mathrm{~F}-\mathrm{FDG}$, and $60-90$ min postinjection of $15 \mathrm{mCi}$ of ${ }^{11} \mathrm{C}-\mathrm{PiB}$. All images were corrected for attenuation, scatter and decay, and smoothed for uniform resolution ${ }^{52}$.

Two-year follow-up volumetric MRI and FDG-PET scans were performed on 17 POST women (age at baseline scan: $57 \pm 3$ years; time to follow-up: $2.2 \pm 0.4$ years) and 12 males within the same age range (age at baseline: $56 \pm 4$ years, time to follow-up: $2.4 \pm 0.4$ years) using the same protocol and equipment as the baseline exams.

\section{Multiparametric Mapping}

All images were processed in Statistical Parametric Mapping (SPM12) implemented in Matlab 7.8, using a fully automated image processing pipeline ${ }^{9-11}$. For each participant, scans were co-registered to the T1MRI and to each other using the Normalized Mutual Information routine ${ }^{53}$. MR scans were processed with 
voxel-based morphometry (VBM), including Jacobian modulation to restore GMV and WMV using the unified segmentation algorithm, DARTEL normalization of the segments, and application of an $8 \mathrm{~mm}$ fullwidth at half maximum (FWHM) smoothing kerne ${ }^{53}$. Co-registered DTI, ASL, and PET scans were spatially normalized using subject-specific transformation matrices obtained from the corresponding structural MRI and smoothed at 10-mm FWHM. For longitudinal analysis, each participant's MRIs were processed using longitudinal routines incorporating rigid-body registration, intensity inhomogeneity correction, and nonlinear diffeomorphic registration ${ }^{53}$. Co-registered follow-up PET were processed using the baseline MRI as the anchor.

ATP/PCr and PiB uptake were quantified using FreeSurfer 6.0 and Desikan-Killiany Atlas-based cortical ROIs ${ }^{54,55}$ applied to the aligned T1-MRI. For PiB analysis, we created an AD-mask by averaging parietal, temporal, frontal, posterior cingulate and precuneus $\mathrm{ROIs}{ }^{56,57}$. PiB uptake in AD-mask was normalized to cerebellar GM uptake, obtained via FreeSurfer. PiB scans were also classified as positive or negative for amyloidosis based on a mean AD-mask standardized uptake value ratio (SUVR) $>1.42^{56,57}$.

\section{Statistical Analysis}

Analyses were performed in SPSS v.25 (IBM) and SPM12. Clinical, demographic and cognitive measures were examined with General Linear Models, t-tests, or Chi-squared tests at $p<0.05$.

We performed several analyses to address age differences between POST and PRE groups ${ }^{11,58}$ : (a) we used box plots and frequency diagrams to ensure that we had sufficient age overlap among women of different MT statuses (PRE: 40-53 years, PERI: 41-60, POST: 46-64 years). This enabled us to examine the effects of endocrine aging separately from those of chronological aging; (b) we included age as a covariate in all analyses; (c) we compared each MT group to age-matched male groups based on the age range of each MT group (MALE $\mathrm{PRE}_{\mathrm{P},}$ MALE $\mathrm{PERI}_{\mathrm{PE}}, \mathrm{MALE}_{\mathrm{POST}}$ ); and (d) we compared 2-year MRI and FDG-PET changes in a subset of POST women and MALE $E_{\text {POST }}$.

All images were analyzed using SPM12, except for MRS and PiB-PET ROI data which were examined using SPSS, as described below. SPM12 analyses were adjusted by age, APOE-4 status, and modalityspecific confounds, e.g. GMV, WMV and FA were adjusted by total intracranial volume (TIV) obtained via Freesurfer, CMRglc by global metabolic activity, CBF by global CBF. Statistical maps were conservatively obtained by first applying an a priori masking image including regions involved in the brain estrogen network with known cognitive functions ${ }^{1}$, and then a stringent cluster-level FWE correction at $p<0.05$, with cluster extent $\geq 20$ voxels. The masking image comprised anterior and posterior cingulate cortex; fusiform gyrus; inferior and medial orbitofrontal cortex; inferior, medial, middle and superior frontal cortex; inferior, middle, and superior temporal gyrus; inferior and superior parietal lobule; insula; medial temporal lobe (amygdala, hippocampus, and parahippocampal gyrus); putamen; precuneus; and thalamus. For WM analysis, the brainstem was also included. Further, results were examined after application of an $a$ priori GM or WM mask to restrict analysis to GM or WM fiber voxels, respectively. Anatomical location of 
GM regions showing group effects was described using MNI coordinates. Identification of clusters in WM tracts was made by reference to the Johns Hopkins University White-Matter Labels atlas ${ }^{59}$.

\section{Biomarker differences between MT groups}

For SPM12 analyses, we used full factorial models to test for biomarker differences between MT groups. If a significant main effect was found, we then proceeded to separately examine the directionality of biomarker differences between paired groups using post-hoc $t$-contrasts.

For ROI analyses, we used mGLMs with post-hoc Sidak tests to test for group differences in ATP/PCr in regions showing MT effects on CMRglc and $C B F$, at $p<0.05$. All analyses were adjusted by age and APOE-4 status as covariates. We used GLMs with post-hoc Sidak tests to examine AD-mask PiB uptake for MT group effects, and for APOE-4 effects by (i) examining APOE-4 status as a covariate; (ii) testing for interactions between MT and APOE-4 status; and (iii) for MT effects within each APOE-4 subgroup, at $p<$ 0.05 . Chi Square tests were used to compare the frequency of PiB-positive scans between MT groups at $\mathrm{p}<0.05$.

\section{Comparisons to age-matched males}

For SPM12 analyses, we used two-sample t-tests to compare each MT group to the corresponding agematched male group, using the same confounders as above. Our main endpoint was detection of sexrelated biomarker differences within implicit masks of the brain regions showing MT stage effects. Our secondary endpoint was detection of group differences in the entire search volume above.

For ROI analyses, we used mGLMs with post-hoc Sidak tests to test for ATP/PCr group differences in regions showing CMRglc and CBF differences between POST and MALE $E_{P O S T}$, at $p<0.05$. We used GLMs with post-hoc Sidak to examine AD-mask PiB uptake for group effects and for APOE-4 status effects (as described above), at $p<0.05$.

\section{Biomarker changes post-menopause}

We investigated whether the structural and CMRglc effects of menopause were persistent 2-years after the first examination in a subset of POST and MALE $E_{\text {POST }}$. SPM12 was applied to extract voxel-based changes in GMV, WMV, and CMRglc by means of one-sample $t$-tests, for each group. The maps of change were then compared between groups. Our main endpoint was detection of biomarker changes within implicit masks of the regions showing MT-stage effects. Our secondary endpoint was detection of longitudinal differences in the entire search volume. Results were examined at $p<0.05$, cluster-level FWE corrected, adjusting for time to follow-up and modality-specific confounds.

\section{Associations between biomarkers and cognitive performance}

Since cognition was preserved post-menopause, we tested for associations between cognitive measures and biomarkers showing positive effects in the POST group, e.g. GMV in precuneus, and CBF and 
ATP/PCr in temporo-parietal regions. Spearman's Rho tests were used to test for correlations with global cognition, and Pearson's $r$ tests for correlations with memory scores, at $p<0.05$, 2-tailed.

\section{Declarations}

\section{ACKNOWLEDGMENTS}

This study was supported by grants from NIH/NIA (P01AG026572, R01AG057931, R01AG035137, R01AG13616), NIH/NCATS UL1TR002384, the Cure Alzheimer's Fund, Maria Shriver's Women's Alzheimer's Movement, and the generous support of Harold W. McGraw III and Nancy G. McGraw. None of the funding organizations or sponsors were involved in study design; in the collection, analysis, or interpretation of data; in writing of the report; or in the decision to submit the article for publication.

\section{AUTHOR CONTRIBUTIONS}

Conceived the idea and planned the research (LM, RBD); contributed to data collection (JD, ES, SJ, LL, GJ, $A R, H H, S P, R I)$; conceptually contributed to the design of experiments (LM, VB, JD, RBD); participated in data analysis (LM, VB, JD, RA, DM) and data interpretation (LM, VB, JD, OE, CG, MdL, RBD); wrote a/o revised the manuscript ( $L M, V B, D M, C G, M d L, R B D)$. All authors reviewed the manuscript.

\section{Competing interests}

The authors declare no competing financial interests.

\section{Data Availability}

The datasets analyzed during the current study can be made available from the corresponding author on reasonable request.

\section{References}

1. Brinton, R. D., Yao, J., Yin, F., Mack, W. J. \& Cadenas, E. Perimenopause as a neurological transition state. Nat Rev Endocrinol. 11, 393-405 https://doi.org/10.1038/nrendo.2015.82 (2015).

2. Monteleone, P., Mascagni, G., Giannini, A., Genazzani, A. R. \& Simoncini, T. Symptoms of menopause - global prevalence, physiology and implications. Nature Reviews Endocrinology. 14, 199-215 https://doi.org/10.1038/nrendo.2017.180 (2018).

3. McEwen, B. S., Alves, S. E., Bulloch, K. \& Weiland, N. G. Ovarian steroids and the brain: implications for cognition and aging. Neurology. 48, 8-15 (1997). 
4. Yue, X. et al. Brain estrogen deficiency accelerates Abeta plaque formation in an Alzheimer's disease animal model. Proc Natl Acad Sci U S A. 102, 19198-19203 https://doi.org/10.1073/pnas.0505203102 (2005).

5. Yao, J. et al. Mitochondrial bioenergetic deficit precedes Alzheimer's pathology in female mouse model of Alzheimer's disease. Proc Natl Acad Sci U S A. 106, 14670-14675 https://doi.org/10.1073/pnas.0903563106 (2009).

6. Comasco, E., Frokjaer, V. G. \& Sundström-Poromaa, I. Functional and molecular neuroimaging of menopause and hormone replacement therapy. Front Neurosci. 8, 388 https://doi.org/10.3389/fnins.2014.00388 (2014).

7. Maki, P. M. The timing of estrogen therapy after ovariectomy-implications for neurocognitive function. Nature Clinical Practice Endocrinology \& Metabolism 4, 494+(2008).

8. Bertram, L. \& Tanzi, R. E. Thirty years of Alzheimer's disease genetics: the implications of systematic meta-analyses. Nat Rev Neurosci. 9, 768-778 https://doi.org/10.1038/nrn2494 (2008).

9. Mosconi, L. et al. Increased Alzheimer's risk during the menopause transition: A 3-year longitudinal study. PloS one. 13, e0207885 (2018).

10. Mosconi, L. et al. Sex differences in Alzheimer risk Brain imaging of endocrine vs chronologic aging. Neurology. 89, 1382-1390 (2017).

11. Rahman, A. et al. Sex-driven modifiers of Alzheimer risk. Neurology. 95, e166 https://doi.org/10.1212/WNL.0000000000009781 (2020).

12. Le Bihan, D. et al. Diffusion tensor imaging: concepts and applications. J Magn Reson Imaging. 13, 534-546 https://doi.org/10.1002/jmri.1076 (2001).

13. Rahman, A. et al. Sex and Gender Driven Modifiers of Alzheimer's: The Role for Estrogenic Control Across Age, Race, Medical, and Lifestyle Risks. Frontiers in Aging Neuroscience. 11, https://doi.org/10.3389/fnagi.2019.00315 (2019).

14. Deecher, D. C. \& Dorries, K. Understanding the pathophysiology of vasomotor symptoms (hot flushes and night sweats) that occur in perimenopause, menopause, and postmenopause life stages. Arch Womens Ment Health. 10, 247-257 https://doi.org/10.1007/s00737-007-0209-5 (2007).

15. Rossmanith, W. G. \& Ruebberdt, W. What causes hot flushes? The neuroendocrine origin of vasomotor symptoms in the menopause. Gynecol Endocrinol. 25, 303-314 https://doi.org/10.1080/09513590802632514 (2009).

16. Cavanna, A. E. \& Trimble, M. R. The precuneus: a review of its functional anatomy and behavioural correlates. Brain. 129, 564-583 https://doi.org/10.1093/brain/awl004 (2006).

17. Hoekzema, E. et al. Pregnancy leads to long-lasting changes in human brain structure. Nature Neuroscience. 20, 287-296 https://doi.org/10.1038/nn.4458 (2017).

18. Gross, C. G. Neurogenesis in the adult brain: death of a dogma. Nature Reviews Neuroscience. 1, 6773 https://doi.org/10.1038/35036235 (2000). 
19. Brunton, P. J. \& Russell, J. A. The expectant brain: adapting for motherhood. Nat Rev Neurosci. 9, 1125 https://doi.org/10.1038/nrn2280 (2008).

20. Kanaan, R. A. et al. Gender differences in white matter microstructure. PLoS One. 7, e38272 https://doi.org/10.1371/journal.pone.0038272 (2012).

21. Bava, S. et al. Sex differences in adolescent white matter architecture. Brain Res. 1375, 41-48 https://doi.org/10.1016/j.brainres.2010.12.051 (2011).

22. Chan, R. W. et al. Structural and Functional Brain Remodeling during Pregnancy with Diffusion Tensor MRI and Resting-State Functional MRI. PLOS ONE. 10, e0144328 https://doi.org/10.1371/journal.pone.0144328 (2015).

23. Aanerud, J. et al. Brain energy metabolism and blood flow differences in healthy aging. Journal of Cerebral Blood Flow and Metabolism(2012).

24. Iadecola, C. Neurovascular regulation in the normal brain and in Alzheimer's disease. Nature Reviews Neuroscience. 5, 347-360 https://doi.org/10.1038/nrn1387 (2004).

25. Masamoto, K. New Horizons in Neurovascular Coupling: A Bridge BetweenBrain Circulation and Neural Plasticity. 1st Edition edn, Vol. 225(Elsevier Science, 2016).

26. Brinton, R. D. The healthy cell bias of estrogen action: mitochondrial bioenergetics and neurological implications. Trends Neurosci. 31, 529-537 https://doi.org/10.1016/j.tins.2008.07.003 (2008).

27. Ding, F., Yao, J., Rettberg, J. R., Chen, S. \& Brinton, R. D. Early decline in glucose transport and metabolism precedes shift to ketogenic system in female aging and Alzheimer's mouse brain: implication for bioenergetic intervention. PLoS One. 8, e79977 https://doi.org/10.1371/journal.pone.0079977 (2013).

28. Mosconi, L. et al. Perimenopause and emergence of an Alzheimer's bioenergetic phenotype in brain and periphery. PloS one. 12, e0185926 (2017).

29. Wang, Y. et al. Midlife Chronological and Endocrinological Transitions in Brain Metabolism: System Biology Basis for Increased Alzheimer's Risk in Female Brain. Sci Rep. 10, 8528 https://doi.org/10.1038/s41598-020-65402-5 (2020).

30. Maki, P. M. \& Henderson, V. W. Cognition and the menopause transition. Menopause. 23, 803-805 https://doi.org/10.1097/gme.0000000000000681 (2016).

31. Rentz, D. M. et al. Sex differences in episodic memory in early midlife: impact of reproductive aging. Menopause. 24, 400-408 (2017).

32. Riedel, B. C., Thompson, P. M., Brinton, R. D. \& Age APOE and sex: Triad of risk of Alzheimer's disease. J Steroid Biochem Mol Biol. 160, 134-147 https://doi.org/10.1016/j.jsbmb.2016.03.012 (2016).

33. Sperling, R. A., Karlawish, J. \& Johnson, K. A. Preclinical Alzheimer disease-the challenges ahead. Nature reviews. Neurology. 9, 54-58 https://doi.org/10.1038/nrneurol.2012.241 (2013).

34. Ferretti, M. T. et al. Sex differences in Alzheimer disease - the gateway to precision medicine.Nature Reviews Neurology(2018). 
35. Levine, M. E. et al. Menopause accelerates biological aging. Proceedings of the National Academy of Sciences 113, 9327-9332, doi:10.1073/pnas.1604558113 (2016).

36. Singh, P. P., Singh, M. \& Mastana, S. S. APOE distribution in world populations with new data from India and the UK. Annals of Human Biology. 33, 279-308 https://doi.org/10.1080/03014460600594513 (2006).

37. Small, G. W. et al. Memory self-appraisal in middle-aged and older adults with the apolipoprotein E-4 allele. The American Journal of Psychiatry. 156, 1035-1038 (1999).

38. Morrison, J. H., Brinton, R. D., Schmidt, P. J., Gore, A. C. \& Estrogen Menopause, and the Aging Brain: How Basic Neuroscience Can Inform Hormone Therapy in Women. The Journal of Neuroscience. 26, 10332-10348 https://doi.org/10.1523/jneurosci.3369-06.2006 (2006).

39. Lobo, R. A. Hormone-replacement therapy: current thinking. Nature Reviews Endocrinology. 13, 220231 https://doi.org/10.1038/nrendo.2016.164 (2017).

40. Miller, V. M. et al. The Kronos Early Estrogen Prevention Study (KEEPS): what have we learned? Menopause 26, 1071-1084, doi:10.1097/gme.0000000000001326 (2019).

41. Henderson, V. W. et al. Cognitive effects of estradiol after menopause: a randomized trial of the timing hypothesis. Neurology. 87, 699-708 (2016).

42. Rocca, W. A., Grossardt, B. R. \& Shuster, L. T. Oophorectomy, estrogen, and dementia: a 2014 update. Molecular and cellular endocrinology. 389, 7-12 (2014).

43. Zeydan, B. et al. Association of Bilateral Salpingo-Oophorectomy Before Menopause Onset With Medial Temporal Lobe Neurodegeneration. JAMA Neurology. 76, 95-100 https://doi.org/10.1001/jamaneurol.2018.3057 (2019).

44. Harlow, S. D. et al. Executive summary of the Stages of Reproductive Aging Workshop + 10: addressing the unfinished agenda of staging reproductive aging. Menopause. 19, 387-395 https://doi.org/10.1097/gme.0b013e31824d8f40 (2012).

45. Pollycove, R., Naftolin, F. \& Simon, J. A. The evolutionary origin and significance of menopause. Menopause. 18, 336-342 https://doi.org/10.1097/gme.0b013e3181ed957a (2011).

46. Hawkes, K., O'Connell, J. F., Jones, N. G., Alvarez, H. \& Charnov, E. L. Grandmothering, menopause, and the evolution of human life histories. Proc Natl Acad Sci U S A. 95, 1336-1339 https://doi.org/10.1073/pnas.95.3.1336 (1998).

47. Takahashi, M., Singh, R. S. \& Stone, J. A Theory for the Origin of Human Menopause. Front Genet. 7, 222 https://doi.org/10.3389/fgene.2016.00222 (2016).

48. Wang, J. et al. Arterial transit time imaging with flow encoding arterial spin tagging (FEAST). Magn Reson Med. 50, 599-607 https://doi.org/10.1002/mrm.10559 (2003).

49. Rijpma, A., van der Graaf, M., Meulenbroek, O., Olde Rikkert, M. G. M. \& Heerschap, A. Altered brain high-energy phosphate metabolism in mild Alzheimer's disease: A 3-dimensional 31P MR spectroscopic imaging study. Neurolmage: Clinical. 18, 254-261 https://doi.org/10.1016/j.nicl.2018.01.031 (2018). 
50. Du, F. et al. Tightly coupled brain activity and cerebral ATP metabolic rate. Proceedings of the National Academy of Sciences 105, 6409-6414, doi:10.1073/pnas.0710766105 (2008).

51. Pettegrew, J. W. et al. Correlation of phosphorus-31 magnetic resonance spectroscopy and morphologic findings in Alzheimer's disease. Arch Neurol. 45, 1093 (1988).

52. Joshi, A., Koeppe, R. A. \& Fessler, J. A. Reducing between scanner differences in multi-center PET studies. Neurolmage. 46, 154-159 https://doi.org/10.1016/j.neuroimage.2009.01.057 (2009).

53. Ashburner, J. \& Friston, K. J. Voxel-based morphometry-the methods. Neurolmage. 11, 805-821 https://doi.org/10.1006/nimg.2000.0582 (2000).

54. Desikan, R. S. et al. An automated labeling system for subdividing the human cerebral cortex on MRI scans into gyral based regions of interest. Neurolmage. 31, 968-980 https://doi.org/10.1016/j.neuroimage.2006.01.021 (2006).

55. Fischl, B. \& FreeSurfer Neurolmage 62, 774-781, doi:10.1016/j.neuroimage.2012.01.021 (2012).

56. Vlassenko, A. G. et al. Imaging and cerebrospinal fluid biomarkers in early preclinical alzheimer disease. Annals of neurology. 80, 379-387 https://doi.org/10.1002/ana.24719 (2016).

57. Mintun, M. A. et al. [11C]PIB in a nondemented population: potential antecedent marker of Alzheimer disease. Neurology. 67, 446-452 https://doi.org/10.1212/01.wnl.0000228230.26044.a4 (2006).

58. Becker, J. B. et al. Strategies and Methods for Research on Sex Differences in Brain and Behavior. Endocrinology. 146, 1650-1673 https://doi.org/10.1210/en.2004-1142 (2005).

59. Hua, K. et al. Tract probability maps in stereotaxic spaces: analyses of white matter anatomy and tract-specific quantification. Neuroimage. 39, 336-347 https://doi.org/10.1016/j.neuroimage.2007.07.053 (2008).

\section{Figures}


a
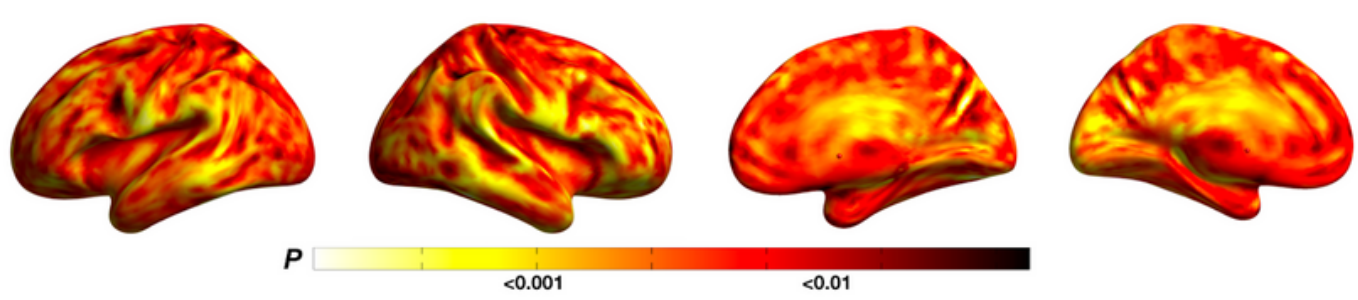

b

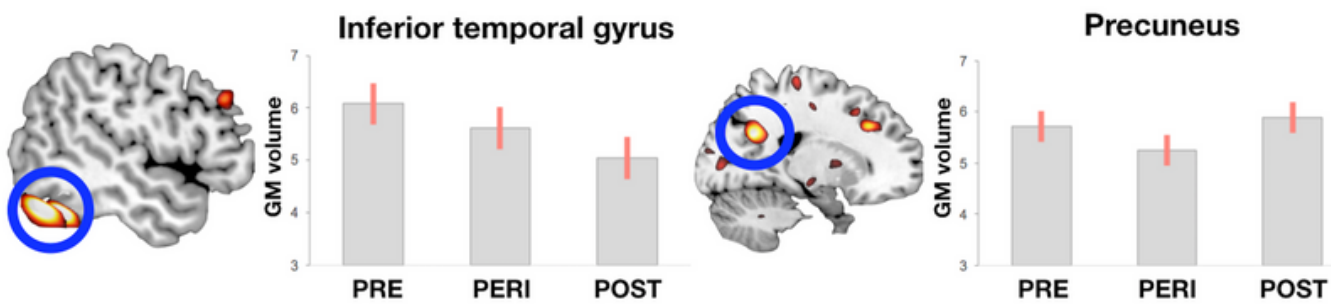

C
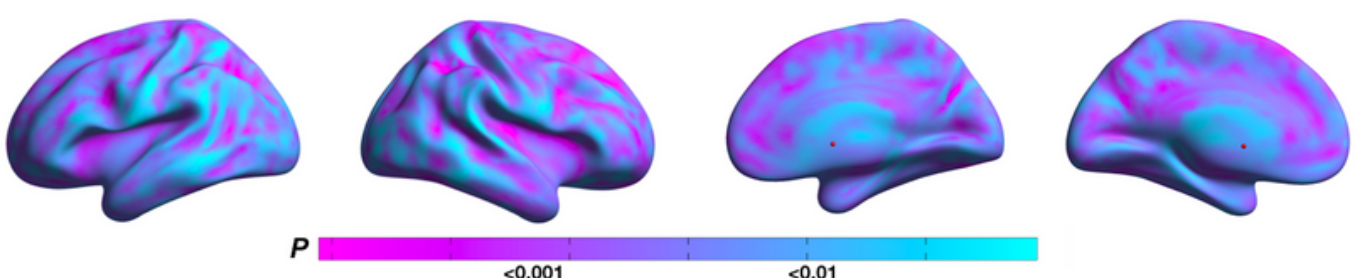

d
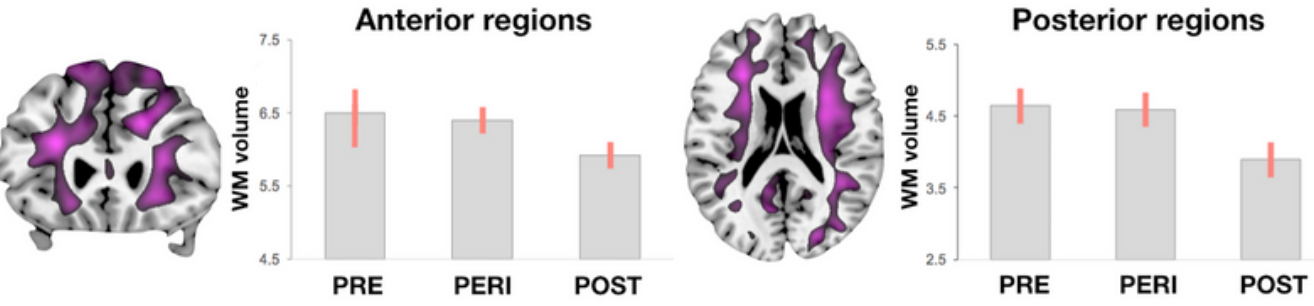

e
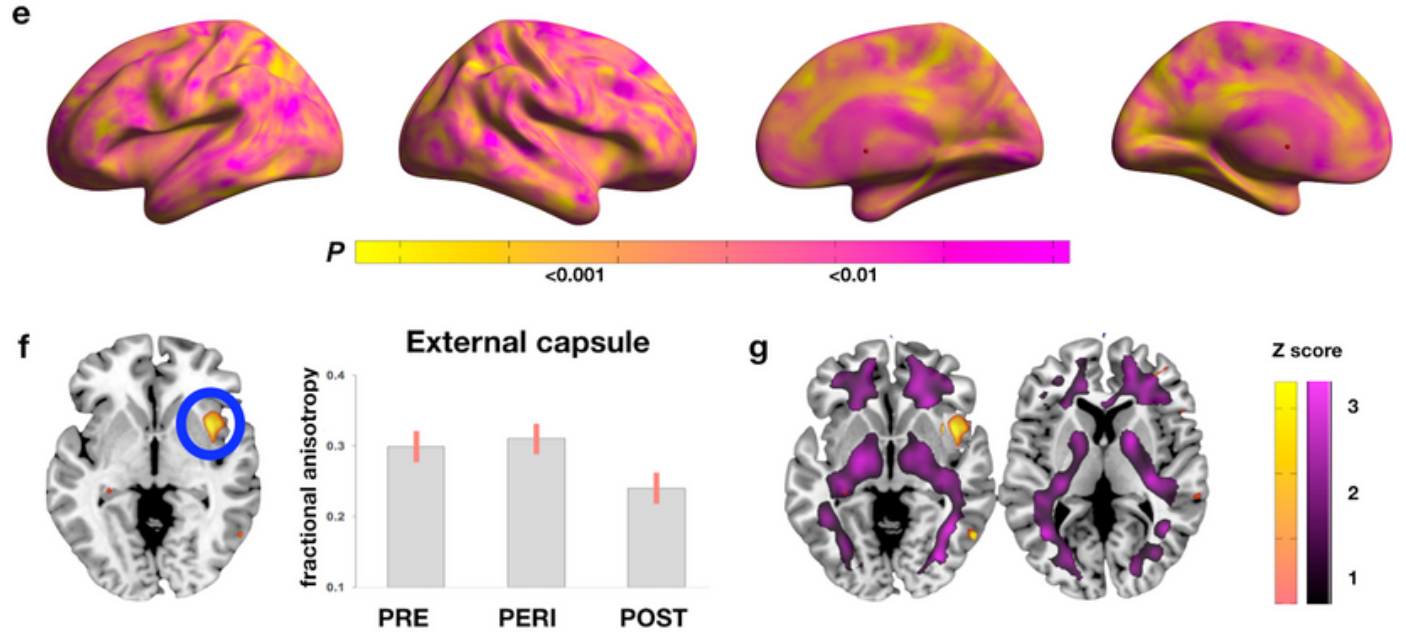

g

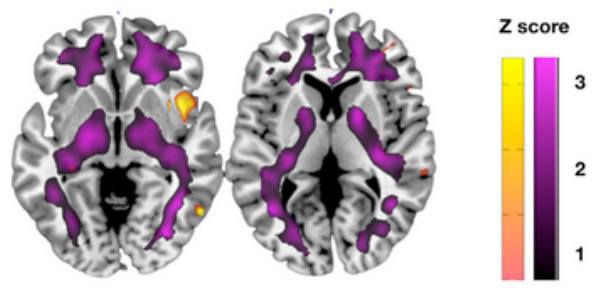

\section{Figure 1}

Structural biomarker differences between MT stage groups. (a) Surface maps of regional gray matter volume (GMV) differences between MT groups at a GM-masked threshold of $p<0.05$, cluster-level corrected for Family-Type Wise Error (FWE), adjusted for age and total intracranial volume (TIV). (b) MRI slice overlays and plots representing age- and TIV-adjusted mean (+SEM) GMV extracted from inferior temporal gyrus and precuneus at the peak of statistical significance. (c) Surface maps of regional white 
matter volume (WMV) differences between MT groups, at a WM-masked threshold of $p<0.05$, cluster-level FWE corrected, adjusted for age and TIV. (d) MRI slice overlays and plots representing age- and TIVadjusted mean (+SEM) WMV extracted from anterior and posterior areas at the peak of statistical significance and averaged between hemispheres. (e) Surface maps of regional DTI-based fractional anisotropy (FA) differences between MT groups at a WM-masked threshold of $p<0.05$, cluster-level FWE corrected, adjusted for age and TIV. (f) MRI slice overlays and plots representing age- and TIV-adjusted mean (+SEM) FA extracted from external capsule at the peak of statistical significance. (g) Slice overlays depicting lack of overlap between WMV (purple) and FA (yellow) effects. Statistical parametric maps (SPMs) are represented on modality-specific color-coded scales with corresponding P values. In (e), Z scores are reported in lieu of $P$ values to enable multi-modality comparison. Anatomical location and statistics for all contrasts are reported in Supporting Information Tables 3-5. Abbreviations: PERI, perimenopause; POST, post-menopause; PRE, pre-menopause. 
a
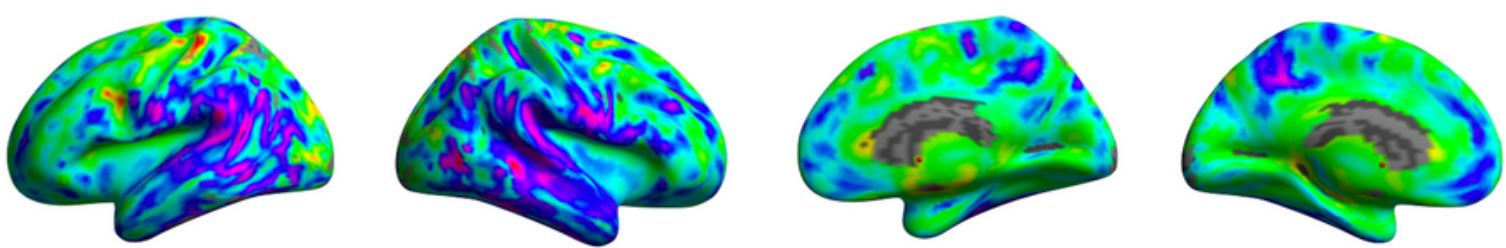

$P$

$<0.001$

$<0.01$

b

Temporal areas
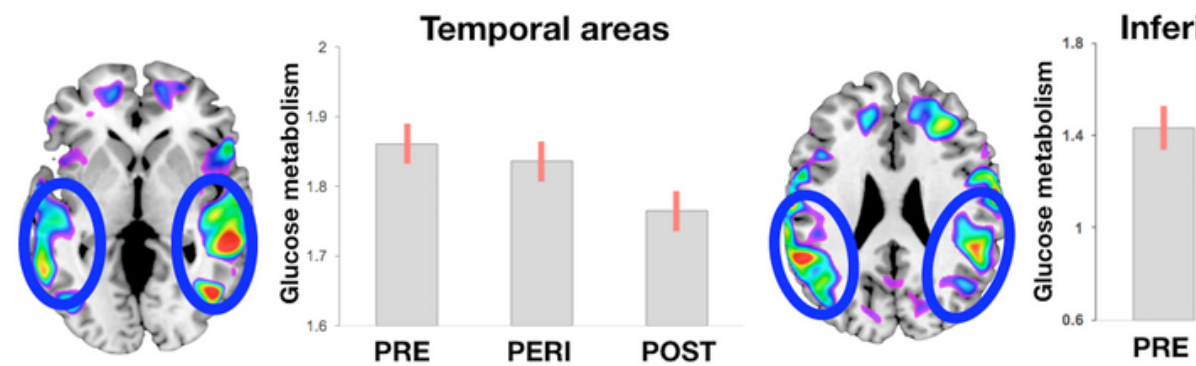

Inferior parietal lobule

C
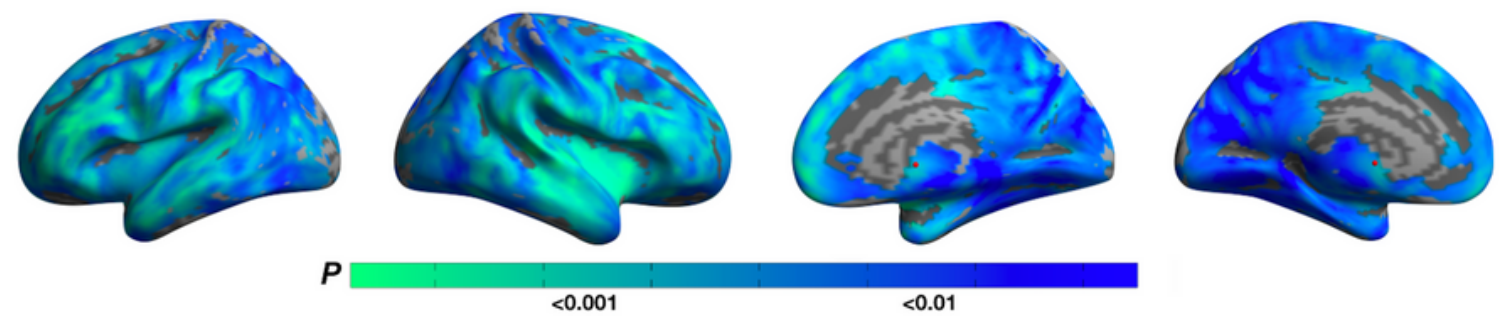

$<0.01$

d
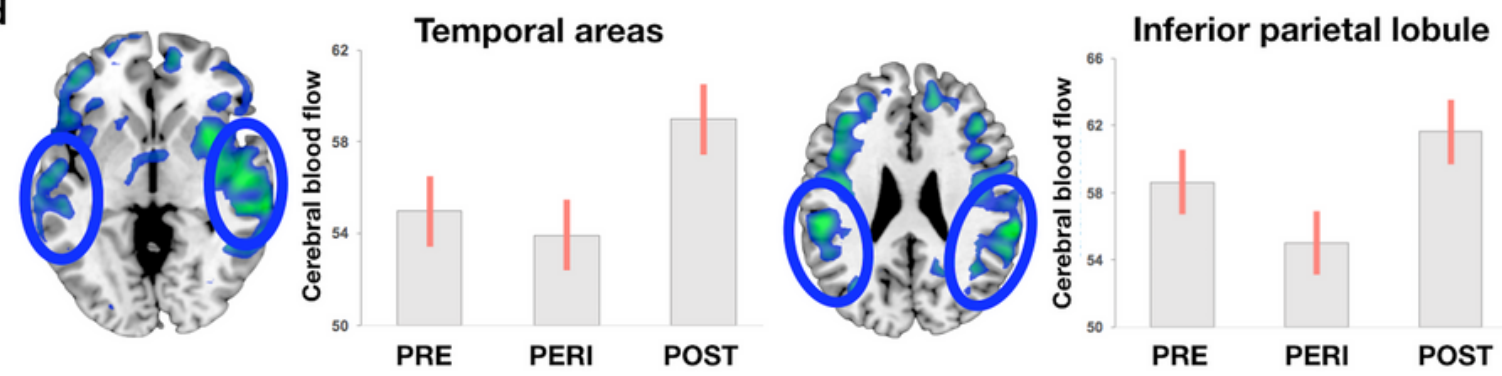

e

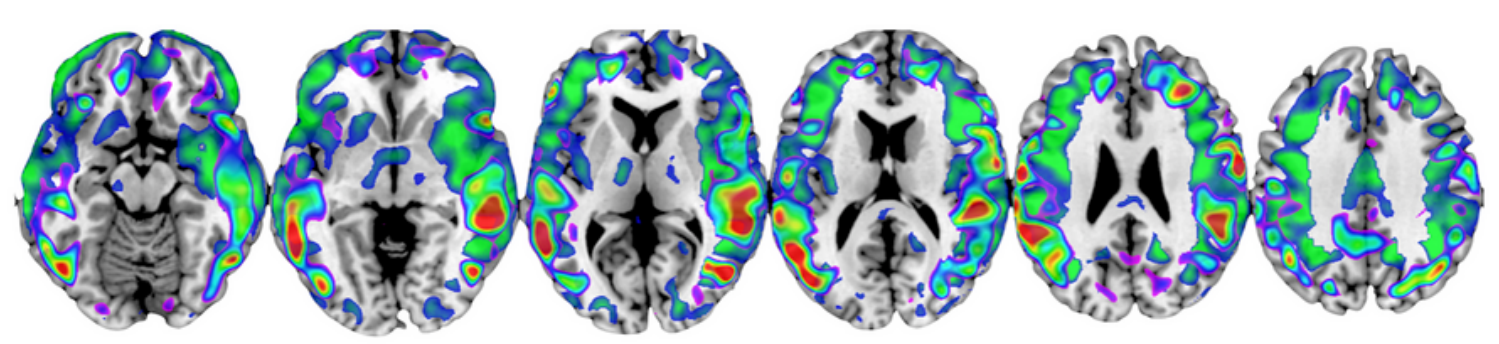

Z score

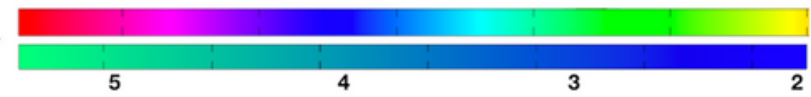

Figure 2

Metabolic biomarker differences between menopausal groups. (a) Surface maps of cerebral glucose metabolism (CMRglc) differences between MT groups, at a GM-masked threshold of $p<0.05$, cluster-level FWE corrected, adjusted for age and global FDG uptake. (b) MRI slice overlays and plots representing age- and global-adjusted mean (+SEM) CMRglc extracted from the temporal and parietal clusters at the peak of statistical significance and averaged between hemispheres. (c) Surface maps of regional cerebral 
blood flow (CBF) differences between MT groups, at a GM-masked threshold of $p<0.05$, cluster-level FWE corrected, adjusted for age and global CBF. (d) MRI slice overlays and plots representing age- and globaladjusted mean (+SEM) CBF extracted from temporal and parietal clusters at the peak of statistical significance and averaged between hemispheres. (e) Slice overlays depicting the overlap between CMRglc (green to red scale) and CBF (blue to green scale) effects. SPMs are represented on modalityspecific color-coded scales with corresponding $P$ values. In (e), Z scores are reported in lieu of $P$ values to enable multi-modality comparison. Anatomical location and statistics for all contrasts are reported in Supporting Information Tables 6 and 7. Abbreviations: see legend to Fig. 1.

POST vs MEN

a

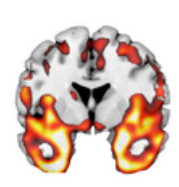

b

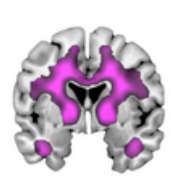

C

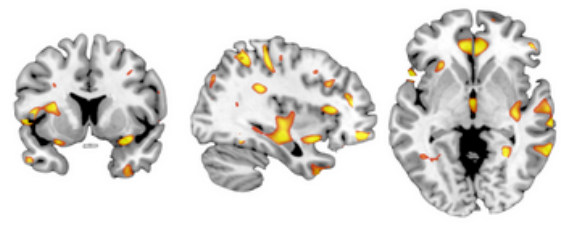

d
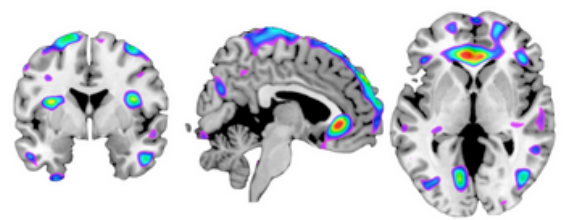

e

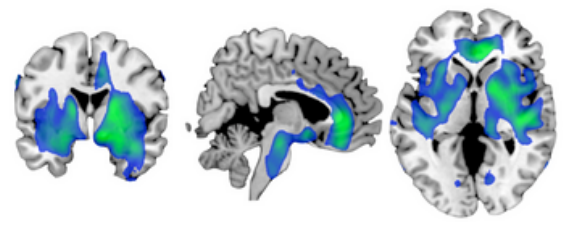

PERI vs MEN
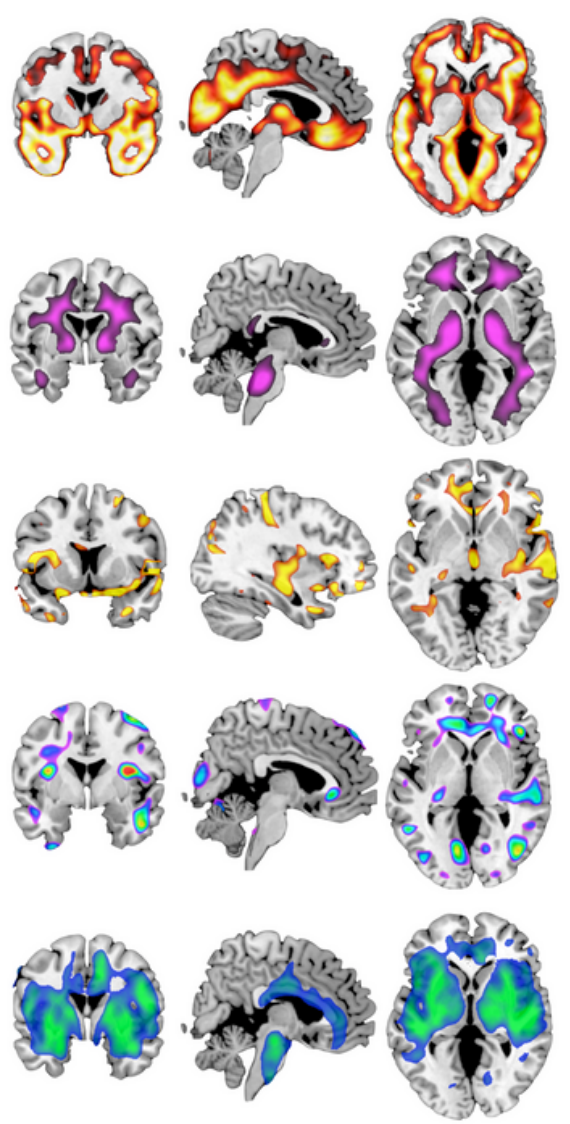

PRE vs MEN
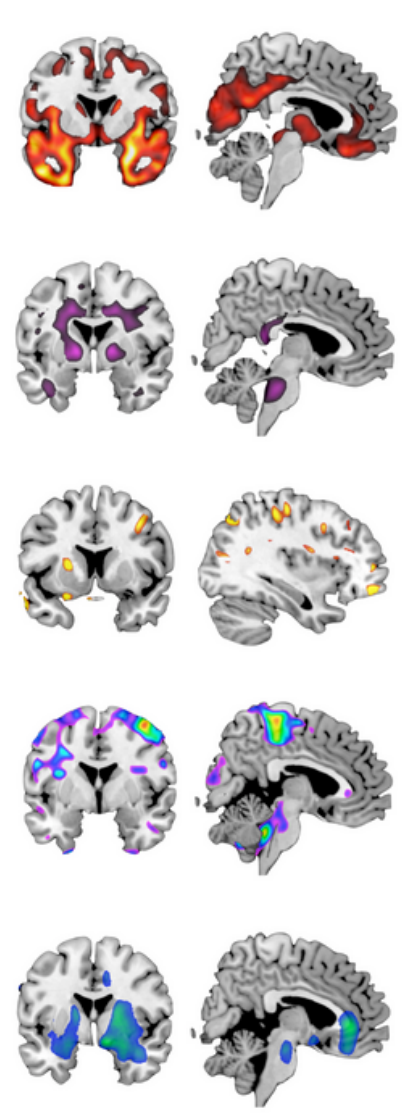
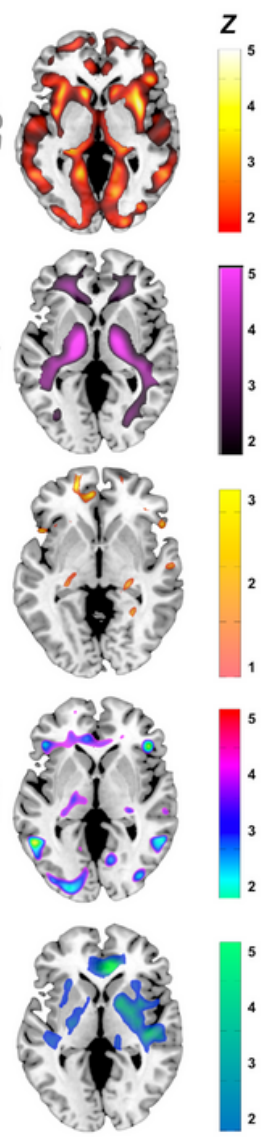

Figure 3

Biomarker differences between each MT stage group and males in the corresponding age ranges. MRI slice overlays displaying: (a) Lower GMV in (left) POST vs. males, (middle) PERI vs. males, and (right) PRE vs. males, at a GM-masked threshold of $p<0.05$, cluster-level FWE corrected, adjusted for TIV. (b) Lower WMV in (left) POST vs. males, (middle) PERI vs. males, and (right) PRE vs. males, at a WM-masked threshold of $p<0.05$, cluster-level FWE corrected, adjusted for TIV. (c) Higher fractional anisotropy (FA) in (left) POST vs. males, (middle) PERI vs. males, and (right) PRE vs. males, at a WM-masked threshold of p<0.05, cluster-level FWE corrected, adjusted for TIV. (d) Lower CMRglc in (left) POST vs. males, (middle) PERI vs. males, and (right) PRE vs. males, at a GM-masked threshold of $p<0.05$, cluster-level FWE corrected, adjusted for age and global CMRglc. (e) Higher CBF in (left) POST vs. males, (middle) PERI vs. 
males, and (right) PRE vs. males, at a GM-masked threshold of $p<0.05$, cluster-level FWE corrected, adjusted for global CBF. SPMs are represented on modality-specific color-coded scales with corresponding Z scores to enable multi-modality comparison. Anatomical location and statistics for all comparisons are reported in Supporting Information Tables 8-12. Abbreviations: see legend to Fig. 1 and 2.

a
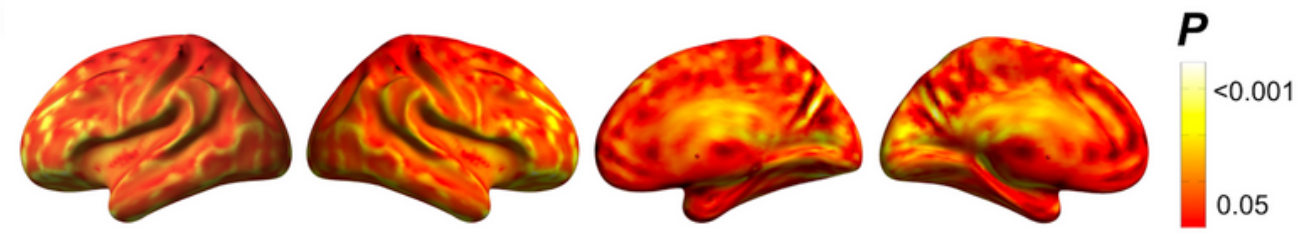

b
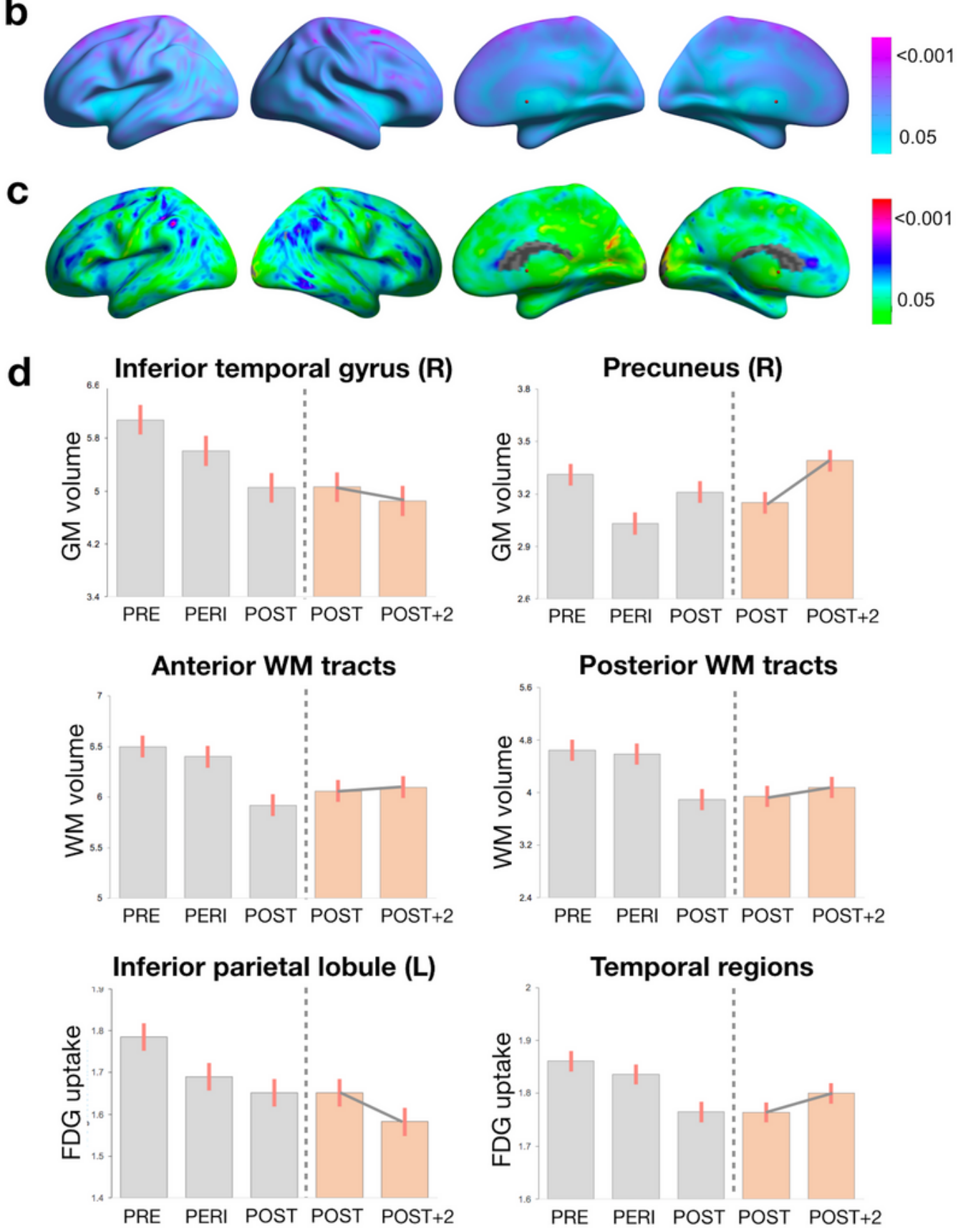

Figure 4 
Biomarker changes between baseline and 2-year follow up in the POST group. (a) Surface maps of GMV change. (b) Surface maps of WMV change. (c) Surface maps of CMRglc change. (d) Plots representing mean signals from the clusters showing effects of interest, comparing [gray] PRE, PERI, and POST groups from the entire cohort; and [orange] baseline (POST) and 2-year follow-up (POST+2) in the subset of 17 POST participants undergoing longitudinal brain scans. SPMs are represented on modality-specific colorcoded scales with corresponding $P$ values. All comparisons are adjusted for time to follow-up and modality-specific confounds. Anatomical location and statistics for longitudinal comparisons are reported in Supporting Information Tables 14 and 15. Unless otherwise specified, biomarker measures are averaged between hemispheres. Abbreviations: $L$, left hemisphere; $R$, right hemisphere.

\section{Supplementary Files}

This is a list of supplementary files associated with this preprint. Click to download.

- SrepSupplementaryInformation011021.docx

- SrepSupplementaryinformationappendix011021.docx 